\title{
Vegetation Database of the Upper Rhine Alluvial Plain Forests
}

\author{
Hans-Gerd Michiels \& Jürgen Kayser
}

Abstract: The Vegetation Database of the Upper Rhine Alluvial Plain Forests (GIVD ID EU-00-009) is a collection of around 1,100 relevés at forest sites from both sides of the Rhein-Valley (Germany and France). The database was filled by different surveys. There are only samples from forests. The data include many parameters of soil and forest site characteristics.

Keywords: forest; France; Germany; site characteristic; soil parameter.

Vegetation Database of the Upper Rhine Alluvial Plain Forests

Scope: - relevés in forest sites of both sides from the Rhein-Valley (Germany and France)

- only samples from forests

- many parameters of soil and forest site

Status: finished

Period: $1994-2004$

Database manager(s): Jürgen Kayser (kayser@idama.de)

Owner: Landesforstverwaltung Baden Württemberg, Germany

Web address: http://www.fva-fr.de

Availability: free upon request

Online upload: no

Online search: yes

Database format(s): MS Access

Export format(s): MS Access

Publication: [NA]

Plot type(s): normal plots

Plot-size range: $25-250 \mathrm{~m}^{2}$

Non-overlapping plots: 1,100

Total plot observations: 1,100

Estimate of existing plots: [NA]

Number of sources: [NA]

\section{Completeness: [NA]}

Valid taxa: 100

Countries: DE: $35.0 \%$; FR: $65.0 \%$

Forest: $100 \%$ - Non-forest: [NA]

Guilds: all vascular plants: $100 \%$

Environmental data: soil depth: 100\%; surface cover other than plants (open soil, litter, bare rock etc.): 100\%; soil pH: $100 \%$; other soil attributes: $100 \%$

Performance measure(s): [NA]

Geographic localisation: GPS coordinates (precision $25 \mathrm{~m}$ or less): $90 \%$; point coordinates less precise than GPS, up to $1 \mathrm{~km}: 10 \%$

Sampling periods: $1990-1999: 77.0 \% ; 2000-2009: 23.0 \%$

Information as of 2012-07-25; further details and future updates available from http://www.givd.info/ID/EU-00-009

Hans-Gerd Michiels (Hans-Gerhard.Michiels@forst.bwl.de)

Forest Ecology, Forest Research Institute Baden-Württemberg, Wonnhalde, 79100 Freiburg, GERMANY

Jürgen Kayser* (kayser@idama.de)

IDaMa GmbH, Rosshaldeweg 4, 79100 Freiburg, GERMANY

*Corresponding author 